\title{
Conservative Treatment for Primary Metatarsalgia
}

\author{
Kentaro Amaha
}

Department of Orthopedic Surgery, Foot and Ankle; St. Luke's International Hospital, Tokyo, Japan

Article Info

\section{Article Notes}

Received: March 19, 2021

Accepted: April 30, 2021

\section{${ }^{*}$ Correspondence:}

${ }^{*}$ Dr. Kentaro Amaha, Department of Orthopedic Surgery, Foot and Ankle; St. Luke's International Hospital, Tokyo, Japan; Telephone No: +81 3 3541-5151; Fax No: 3544-0649; Email: amaken@luke.ac.jp.

${ }^{\circledR} 2021$ Amaha K. This article is distributed under the terms of the Creative Commons Attribution 4.0 International License.

\section{Keywords}

Metatarsalgia

Toe exercise

Conservative treatment

Metatarsal pads

\begin{abstract}
Metatarsalgia is one of the most common causes of forefoot pain, and it is characterized by pain in the front part of the foot under the head of the metatarsal bones. Primary metatarsalgia is idiopathic, but it has been suggested to be related to forefoot plantar compression. Because of the various causes of metatarsalgia, there is the need to thoroughly consider the etiology of metatarsalgia to find novel, effective, and conservative treatments for metatarsalgia to avoid surgical treatment. Pressure reduction or redistribution can be achieved using toe exercise, flat shoe inserts, metatarsal pads, custommolded inserts, and rockerbars. There was no need for one treatment. If toe function was poor, toe exercises were recommended. If dorsiflexion of the ankle joint was limited, the Achilles tendon was stretched. If the pain was localized to the plantar aspect of the 2nd MTP, a decompression insole was applied. If the pain was limited to the plantar aspect of the 2nd MTP, a decompression insole was worn. If swelling occurred, anti-inflammatory drugs were indicated to reduce inflammation. The combination of the two was appropriate for this condition. Toe exercises can improve balance and are worth trying. An in-depth understanding of the various etiologies of metatarsal and toe deformities is essential for successful treatment.
\end{abstract}

\section{Introduction}

Metatarsalgia was first described in 1988 as pain in and around the head of the metatarsal or metatarsophalangeal joint and adjacent soft tissues ${ }^{1}$. It is one of the most common causes of forefoot pain, and it is characterized by pain in the front part of the foot under the head of the metatarsal bones. It is considered a syndrome with primary and secondary causes. Primary metatarsalgia is idiopathic, but it has been suggested to be related to forefoot plantar compression ${ }^{2}$. Secondary metatarsalgia is associated with metabolic, neurological, postsurgical, or traumatic events. Although the exact cause is unclear, the mechanical overload of weight-bearing forces is considered the underlying etiology of primary metatarsalgia ${ }^{4}$. The first approach to treatment is conservative, and it requires more detailed knowledge of the forefoot condition.

\section{Why Conservative Treatment?}

Primary metatarsalgia is regarded as an abnormality related to the anatomy of the metatarsal bones, as well as their relationship with the rest of the foot, and it leads to overload. ${ }^{2}$ Metatarsal bone length discrepancy has received the most attention so far ${ }^{3}$. Our previous study showed that index minus is very common in patients with metatarsalgia. This suggests that the second metatarsal was relatively long, and the metatarsal length seemed to influence the onset of metatarsalgia 4 . Previous studies have shown that metatarsal shortening osteotomy following the failure of nonsurgical 
treatment is an effective modality for the management of metatarsalgia ${ }^{5-7}$. However, this type of osteotomy remains debated. A previous study showed that metatarsal length did not affect plantar-loading parameters ${ }^{8}$. Furthermore, transfer metatarsalgia and non-union following shortening osteotomy are severe problems that cannot be ignored ${ }^{7}$. Therefore, we have to find novel, effective, and conservative treatments for metatarsalgia ${ }^{9}$ to avoid surgical treatment. There is the need to thoroughly consider the etiology of metatarsalgia.

\section{Evolutionary Perspective}

Humans walk upright regularly. From a historical standpoint, human bipedalism evolved from apequadrupedalism. This trait makes us unique among primates. Weidenreich noted that the bipedal condition of man is entirely different from that of other primates in terms of the extension position in the frontal plane ${ }^{10}$. This extreme extension position facilitates quicker and easier lifting and the forward movement of the leg. As a result, this enabled humans to leave the forest, which is the habitat of all other apes. Our feet no longer have the ability to grasp and manipulate objects because the toes became shorter and the big toe grew in line with the other toes. In non-human apes, pedal digital flexors account for a large proportion of the muscle volume crossing the ankle joint. Compared with non-human apes, humans have larger triceps ${ }^{11}$. To bear the weight from this extra muscle volume, humans have evolved to have an enlarged calcaneus. As a result of the bipedal evolution, humans lost the strength of pedal digital flexors and gained strong triceps and heel bones. In addition to the evolutionary weakness of toe plantar flexion, human muscles tended to decrease in mass with age. It is easy to assume that the toes are predisposed to dysfunction, which can lead to pathological conditions of the foot.

\section{Mechanical Basis of Metatarsalgia}

The stance phase accounts for $60 \%$ of the gait cycle, and it is further subdivided into three distinct "rocker" phases (Figure 1). The first rocker phase accounted for $10 \%$ of the total gait cycle. During the subsequent stance phase, the ankle functioned as a second rocker, accounting for $20 \%$ of the gait cycle. During this phase, the foot remained flat on the ground. During the toe-off phase, which is the third and final phase of the rocker that accounts for $30 \%$ of the gait cycle, only the forefoot was in contact with the ground. During the toe-off phase of the gait cycle, the metatarsal heads and toes were the only points of contact with the ground, and they became load-bearing. When walking, the load increases by approximately two times the bodyweight before toe-off at the MTP joints ${ }^{12}$, which is the combined effect of the forward-falling and ground reaction force loads applied to the forefoot. When the toes lose the ability to functionally push off the ground, there



Figure 1: The stance phase is further subdivided into three different rocker phases

is an increased load on the metatarsal area. Repetitive overloading in the metatarsal area causes metatarsalgia. Zelik et al. investigated the coordination of MTP flexors and extensors using electromyography recordings and showed substantial inter-subject variability of the relative magnitudes of MTP flexor activation, even though their studies were on healthy human participants ${ }^{13}$. Agerelated changes and pathological conditions cause further differences in the MTP flexors ${ }^{14}$.

\section{Keys in determining conservative treatment methods}

First, the kind of shoes the patient wears is checked; for example, high heels or flat shoes. Then, the part that is worn out is assessed. If the lateral side is worn out, lateral ground contact was presumed, and an excessive load is applied. The next physical examination is performed to check the range of motion of the ankle joint. If dorsiflexion of the ankle joint is limited, the Achilles tendon (triceps muscle) is contracted, and the forefoot is easily overloaded. We try dorsiflexing the ankle joint separately with the knee joint extended and the knee joint flexed. If dorsiflexion of the ankle joint improves when the knee is flexed, contracture of the gastrocnemius muscle is presumed. Next, the patient performs a flexion movement of the toes, and it is evaluated. In several cases, the patient is unable to flex and extend the toes well, which indicates a toe dysfunction. Next, the patient stands and the neutrality of the hindfoot is assessed. In the case of varus, the foot is grounded on the lateral side, which may put more stress on the 4 th and 5th MTP joints. The location of the greatest tenderness is determined. The most common is the plantar side of the 2nd MTP joint, where the transverse arch collapses and the metatarsals are overloaded based on their length. It is also necessary to observe the condition of the plantar skin, which often forms calluses on the bottom side of the 2 nd MTP joint (Figure 2). This is indicative of excessive loading in the area. After verifying the above, the treatment method is selected. 


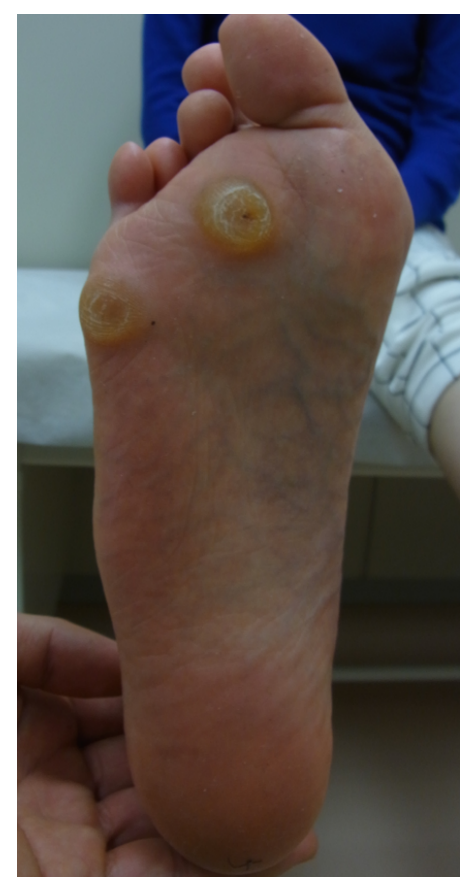

Figure 2: The plantar view of a patient with the formation of callus at the plantar aspect of the foot

\section{Conservative Treatment for Primary Metatarsalgia}

Pressure reduction or redistribution can be achieved using toe exercise, flat shoe inserts, metatarsal pads, custom-molded inserts, and rockerbars.

There was no need for one treatment. If toe function was poor, toe exercises were recommended. If dorsiflexion of the ankle joint was limited, the Achilles tendon was stretched. If the pain was localized to the plantar aspect of the 2nd MTP, a decompression insole was applied. If the pain was limited to the plantar aspect of the 2nd MTP, a decompression insole was worn. If swelling occurred, anti-inflammatory drugs were indicated to reduce inflammation. The combination of the two was appropriate for this condition.

\section{Toe exercise}

We reported that toe exercise improved toe flexion strength and metatarsalgia ${ }^{4}$. Therefore, metatarsalgia may be alleviated by improving toe function. This is consistent with other reports that metatarsalgia was improved by toe training, although this is a case report. Metatarsalgia is thought to be caused by imbalance due to load control, and toe training can be effective treatment ${ }^{15}$. Toe exercises strengthen the intrinsic muscles of the foot and, consequently, contribute to the stability of the foot arch during the toe-off phase ${ }^{16}$. If toe function is poor, the toes cannot push off the ground firmly during the toe-off phase, and the metatarsal area receives loading. By training the toe function, the toes can cover the load on the ball area, reducing the load at each step and improving metatarsalgia.
Toe exercises are safe and effective not only for improving metatarsalgia, but also for improving balance, and they should be attempted for any form of metatarsalgia.

\section{Achilles stretching exercise}

The equinus ankle position due to contracture of the heel cord and calf muscle is a contributing factor to metatarsalgia. Physical therapy focuses on stretching the heel cord and calf muscles to reduce pressure on the forefoot and improve metatarsalgia. Evidence suggests that the mechanical and morphological properties of the Achilles tendon are affected by acute exercise. Prolonged stretching and repeated maximal isometric contractions of the triceps surae appear to cause the most consistent and pronounced reductions in Achilles tendon stiffness and hysteresis ${ }^{17}$.

\section{Shoes and Insoles}

The condition of the shoes should be checked, and they should be changed if necessary. Wearing proper shoes is essential for the treatment of metatarsalgia, especially if the shoes are causing symptoms. Shoes with high heels put more stress on the metatarsal heads. Shoes suitable for the treatment of metatarsalgia include shoes with sufficient cushioning in the sole, a wide toe box, and a low heel. It is important to educate the patient about what kind of shoes are not good for metatarsalgia. Improvements in footwear and orthotics can reduce localized pressure on the foot and relieve pain. Orthotics with metatarsal pads (Figure 3) have been shown to reduce plantar pressure on the metatarsal heads. In a study of insole-induced plantar pressure, the peak pressure reduction curve showed a general trend of decreasing peak pressure with increasing insole thickness. The peak pressure had a maximum reduction of approximately $26.4 \%$ in the insole-only condition $^{18}$.

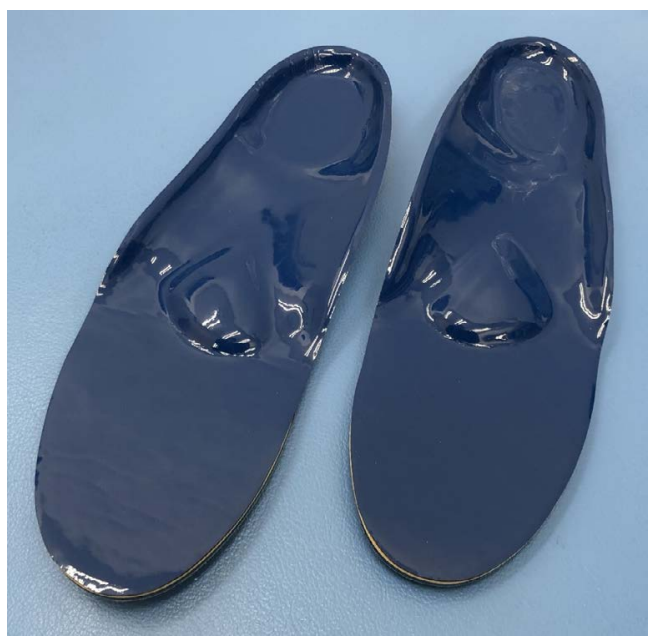

Figure 3: Insole with the metatarsal pad 


\section{Callus Shaving}

If the patient has callus formation on the sole, pain can be improved by shaving with a callus file, pumice stones, a scalpel, or a callus blade. This is a simple and effective method for managing painful conditions.

\section{Surgical Treatment}

Surgery is indicated when pain does not improve after treatment with the above-mentioned therapies. The type of surgery is determined by the underlying etiology. If the problem is the length of the metatarsal bone, a shortening osteotomy is indicated, and if there is contracture of the Achilles tendon, a lengthening procedure is preferred. In some cases, metatarsal head resection or plantar condylectomy may be indicated. These surgical techniques may be performed simultaneously depending on the condition. However, unnecessary surgery should be avoided as much as possible, and a detailed knowledge of the condition is of utmost importance.

\section{Conclusions}

Because of the various causes of metatarsalgia, the initial treatment should be nonoperative. Nonoperative treatment may include wearing appropriate footwear and physical therapy, such as toe exercises and insoles. Toe exercises can improve balance and are worth trying. An in-depth understanding of the various etiologies of metatarsalgia is essential for successful treatment. An indepth understanding of the various etiologies of metatarsal and toe deformities is essential for successful treatment.

\section{Conflict of Interest}

There are no conflicts of interest to declare.

\section{Funding}

None

\section{References}

1. Espinosa N, Maceira E, Myerson MS. Current concept review: metatarsalgia. Foot Ankle Int. 2008; 29(8): 871-879.

2. Reynold JC. Developmental disorders: adult foot Part 2. Metatarsalgia In: Gould, JS, editors. The foot book. Baltimore: Wiliams \& Wilkins; 1988. p. 219-227.
3. Mann RA, Chou LB. Surgical management for intractable metatarsalgia. Foot Ankle Int. 1995; 16(6): 322-327.

4. Amaha K, Arimoto T, Kitamura N. Effect of toe exercises and toe grip strength on the treatment of primary metatarsalgia. J Orthop Surg Res. 2020; 15(1): 580.

5. Khurana A, Kadamabande S, James S, et al. Weil osteotomy: assessment of medium-term results and predictive factors in recurrent metatarsalgia. Foot Ankle Surg. 2011; 17(3): 150-157.

6. Pearce CJ, Calder JD. Metatarsalgia: proximal metatarsal osteotomies. Foot Ankle Clin. 2011; 16(4): 597-608.

7. Schuh R, Trnka HJ. Metatarsalgia: distal metatarsal osteotomies. Foot Ankle Clin. 2011; 16(4): 583-595.

8. Kaipel M, Krapf D, Wyss C. Metatarsal length does not correlate with maximal peak pressure and maximal force. Clin Orthop Relat Res. 2011; 469(4): 1161-1166.

9. Federer AE, Tainter DM, Adams SB, et al. Conservative management of metatarsalgia and lesser toe deformities. Foot Ankle Clin. 2018; 23(1): 9-20.

10. Weidenreich F. Generic, specific and subspecific characters in human evolution. Am J Phys Anthropol. 1946; 4(4): 413-32.

11. Payne RC, Crompton RH, Isler K, et al. Morphological analysis of the hindlimb in apes and humans. I. Muscle architecture. J Anat. 2006; 208(6): 709-724.

12. Al-Munajjed AA, Bischoff JE, Dharia MA, et al. Metatarsal loading during gait-a musculoskeletal analysis. J Biomech Eng. 2016; 138(3): 4032413 .

13. Zelik KE, La Scaleia V, Ivanenko YP, et al. Coordination of intrinsic and extrinsic foot muscles during walking. Eur J Appl Physiol. 2015; 115(4): 691-701.

14. Menz HB. Biomechanics of the Ageing Foot and Ankle: A Mini-Review. Gerontology. 2015; 61(4): 381-388.

15. Yoo WG. Effect of the intrinsic foot muscle exercise combined with interphalangeal flexion exercise on metatarsalgia with Morton's toe. J Phys Ther Sci. 2014; 26(12): 1997-1998.

16. Prentice WE. Rehabilitation techniques in sports medicine. McGrawHill Humanities; 2010.

17. Obst SJ, Barrett RS, Newsham-West R. Immediate effect of exercise on Achilles tendon properties: systematic review. Med Sci Sports Exerc. 2013; 45(8): 1534-44.

18. Chen WM, Lee SJ, Lee PV. Plantar pressure relief under the metatarsal heads: therapeutic insole design using three-dimensional finite element model of the foot. J Biomech. 2015; 48(4): 659-65. 